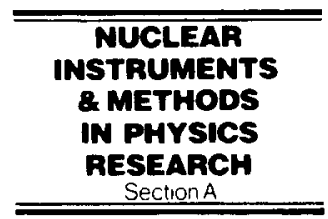

\title{
Design and construction of a thermal neutron target for the RPI linac
}

\author{
Y. Danon, R.C. Block* , R.E. Slovacek \\ Department Of Nuclear Engineerıng And Engineering Phystcs, Rensselaer Polytechnic Institute, Troy, NY 12180, USA
}

Received 12 April 1994

\begin{abstract}
To perform thermal cross section measurements the low energy neutron intensity from the RPI linac facility was increased. A new Enhanced Thermal Target (ETT) was designed, constructed and tested. The thermal flux of the new target was up to six times greater than the flux from the previous RPI Bounce Target (BT). This additional gain allows transmission measurements to be performed in the energy range of 0.001 to $15 \mathrm{eV}$ with high statistical accuracy in a short time $(\sim 40 \mathrm{~h})$. The ETT was also designed to be coupled to a cold moderator that will give an additional flux increase factor of about 9 below $3 \mathrm{meV}$. Design calculations for the cold moderator including neutronics and cryogenics are also given.
\end{abstract}

\section{Introduction}

The RPI linac facility is used for transmission and capture cross section measurements with the time-of-flight (TOF) method. The neutron flux is generated by directing the linac $60 \mathrm{MeV}$ pulsed electron beam at a water-cooled photoneutron tantalum target. Moderation is achieved with a $17-\mathrm{cm}$-diameter by $2.54-\mathrm{cm}$-thick polyethylene disk. The standard target used for over twenty years was named the Bounce Target (BT) because of the particular geometry shown in Fig. 1, where the tantalum photoneutron target is not in the neutron beam line and the moderator is adjacent to the target [1]. However the geometry of the BT is not efficient for neutron moderation and therefore this target could not be used for subthermal-energy experiments. The practical lower energy limit of the BT is about $0.005 \mathrm{eV}$ for transmission measurements. This limit is set by the low flux intensity and poor signal-to-background ratio of the BT which requires a long experiment time to obtain a reasonable statistical error and therefore makes such an experiment expensive and the background correction large.

This limit created the need for a new target that could achieve a higher flux at the thermal region while preserving or improving the signal-to-background ratio. Another requirement fora new design was to have a flux intensity and resolution that will permit measurements in a broad energy range from $0.001 \mathrm{eV}$ to $20 \mathrm{eV}$. The higher energy limit $(20 \mathrm{eV})$ is set by the TOF spectrometer resolution for the $15 \mathrm{~m}$ flight station used. This wide energy range leads to more efficient measurements since only one experiment is required to cover the effective energy range of the $15 \mathrm{~m}$ flight station. When considering experiments in the few

\footnotetext{
* Corresponding author.
}

$\mathrm{meV}$ energy range, acryogenic target becomes an attractive solution for obtaining higher neutron flux. By cooling the moderator, the neutron Maxwellian distribution peak can be shifted to lower energies thus increasing the neutron flux at energies below the room temperature peak. Cooling a moderator to about $30 \mathrm{~K}$ can achieve a practical gain of about 8 to 10 at $1 \mathrm{meV}$ relative to a room temperature moderator.

\section{Target design}

As the first step in designing a new low-energy neutron target for the RPI linac a conceptual design was created. The conceptual design served as the basis for further calculations and is shown in Fig. 2, where the basic elements of the new target are shown. The tantalum target is located in the center of a water moderator and is cooled by water. The water moderator region is shown surrounded by a graphite reflector region. The cold moderator is positioned in front of the water moderator (in the direction of the neutron beam). The cold moderator is surrounded by an evacuated aluminum enclosure and is kept cold by a copper cold finger that conducts heat to a helium cooling head. Also shown is a $\mathrm{Cd}$ de-coupler between the water moderator and the cold moderator; the de-coupler helps narrow the moderated neutron beam time spread and thus improves the spectrometer energy resolution [2].

The design stage was divided into two phases. In the first stage a room-temperature target was designed which can replace the existing BT and improve the flux intensity below $18 \mathrm{eV}$. This target was designed in a way to be coupled to a cold moderator and achieve additional gain below $20 \mathrm{meV}$. Once the room-temperature target was 


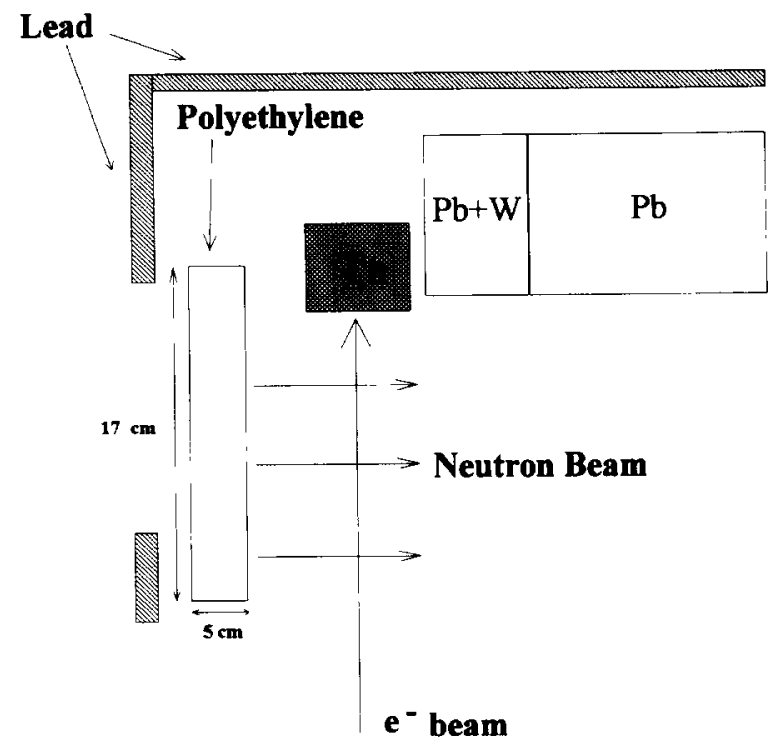

Fig. 1. A sketch of an overhead view of the RPI bounce target.

completed it was tested and sub-thermal cross section measurements were made. These measurements serve to evaluate the need for a cryogenic moderator.

In the second phase the cold moderator was designed and key parameters were quantified. Information on the expected gain and the type of cooling system required is given and provides the data necesary to evaluate the costs and benefits of installing acold moderator.

\section{Room temperature design calculations}

The tool for calculating various geometry configurations was chosen to be the MCNP Monte Carlo code [3]. This code has the ability to simulate three dimensional geometry; it is easy to use, produces results in a short time, and has the ability to simulate the tantalum target neutron source distribution. Another advantage of the MCNP code is the availability of a cross section library that has all the materials needed for this design. The MCNP code also supports time-dependent calculations that are useful when calculating the time-dependent neutron current emitted from the moderator surface. Such a calculation is needed to examine the expected time resolution of a given moderator geometry.

\subsection{Cubic water moderator}

The first step in the design was a calculation for a simple case of an isotropic neutron point source in a cube of water moderator. In this calculation the objective was to find the size of water moderator that maximized the neutron current emerging from the cube face (the term current as used here refers to the fraction of the total number of
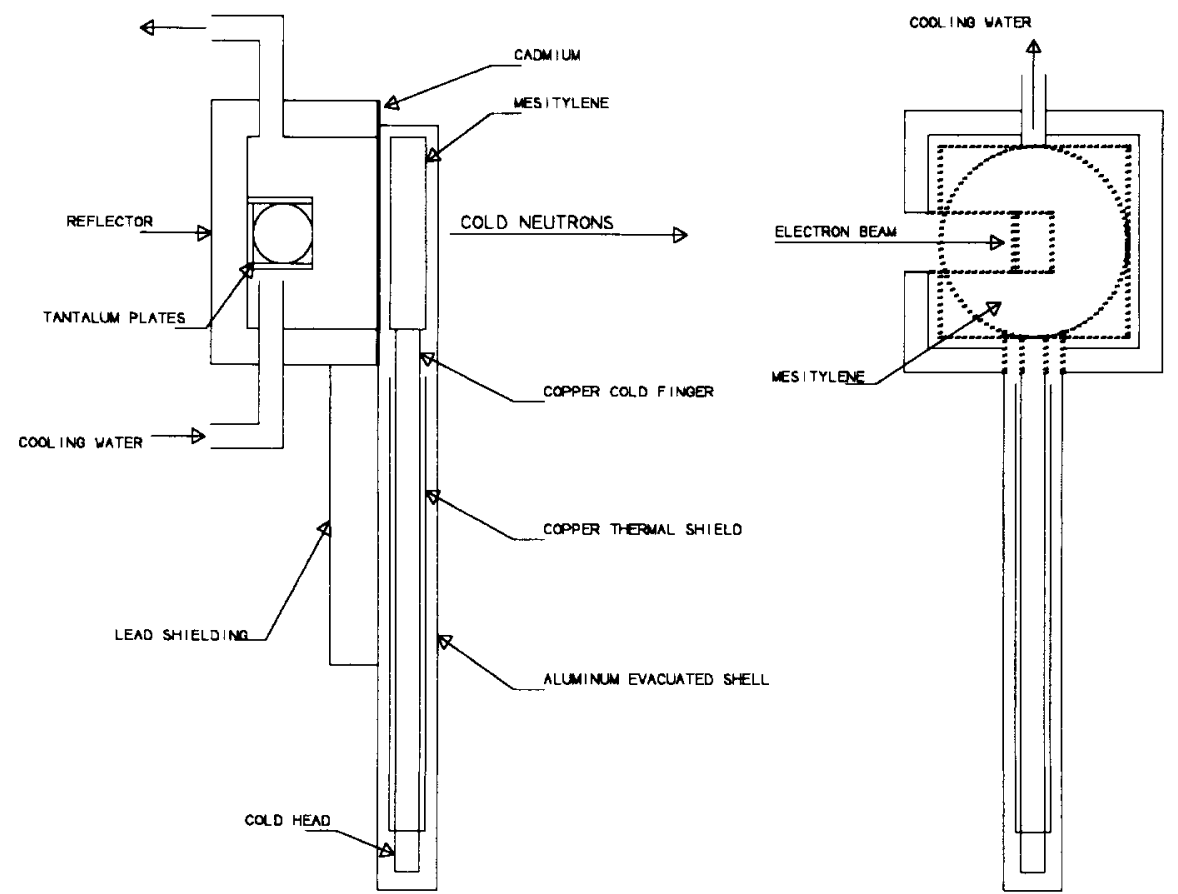

Fig. 2. The enhanced thermal target conceptual design. The figure on the left is a view as seen from the linac while on the right is the view from the flight path. 


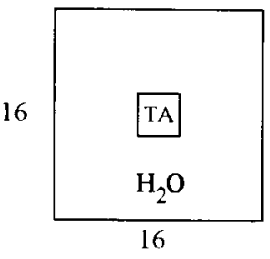

(a)

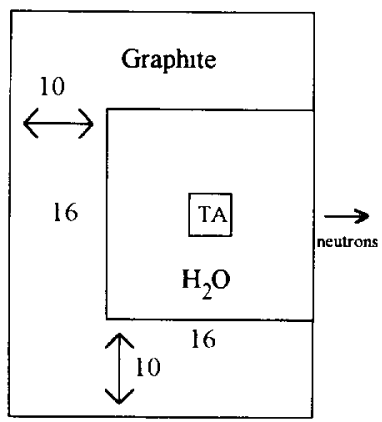

(b)

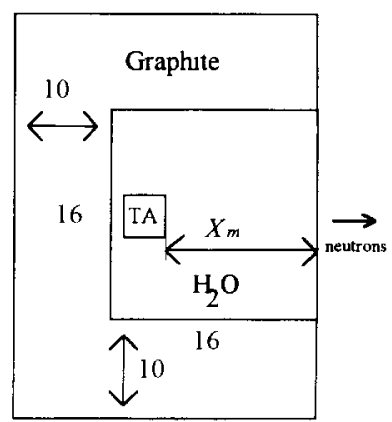

(c)

Fig. 3. MCNP simulation geometry (a) tantalum source in a cube of water, (b) centered tantalum source with a graphite reflector (c) the tantalum source moved closer to the back moderator face. The numbers refer to dimensions in $\mathrm{cm}$.

neutrons crossing a surface in one direction). The neutron source term is an isotropic point source with an evaporation energy spectrum given by the equation

$\phi(E)=C E \mathrm{e}^{-E / T}$,

where $E$ is the neutron energy in $\mathrm{MeV}, T=0.46 \mathrm{MeV}$ is the effective neutron temperature for the tantalum target [4] and $C$ is a normalization constant such that

$\int_{0}^{\infty} \phi(E) \mathrm{d} E=1$.

This gives $C=4.73$ and a mean neutron energy of 0.92 $\mathrm{MeV}$. The results of a series of MCNP caclulations with a variable cube size tallied in the energy bin 0.025 to 0.08 $\mathrm{eV}$ showed that the optimum cube side length is about 16 $\mathrm{cm}$.

\subsection{Reflected geometry}

The next step in the calculation was to introduce the tantalum target material into the water cube and examine the benefits of a reflected geometry. The tantalum target is first represented by a $5 \mathrm{~cm} \times 5 \mathrm{~cm} \times 5 \mathrm{~cm}$ cube of tantalum in the center of the water moderator with an isotropic (in position and direction) neutron source with the energy distribution of Eq. (1). The water cube was then surrounded by a layer of graphite $10 \mathrm{~cm}$ thick; the geometry is shown in Figs. $3 b$ and $3 c$.

The effect of adding a reflector as shown in Fig. $3 \mathrm{~b}$ was found to increase the neutron flux by $25 \%$ relative to the geometry of Fig. 3a. Other reflector materials were also studied and the most effective was found to be beryllium. With beryllium only half the graphite reflector size is needed to achieve the same gain, also when beryllium is placed in the high energy gamma flux it can serve as an additional neutron booster generating neutrons from the $(\gamma, n)$ reaction. Beryllium was not used because it was not readily available, and it is a hazardous material to work with.

The next step in the design was to change the modera- tor geometry to the one shown in Fig. 3c. In this geometry the tantalum target is close to the back face of the water moderator and calculations were done to determine the thickness $X_{\mathrm{m}}$ of water needed in front of the moderator to achieve maximum neutron current in the energy bin of 0.025 to $0.08 \mathrm{eV}$. The motivation for trying this geometry was to check the target performance when using two moderators; one is the water moderator region surrounding the target, and the other is a cold moderator in front of the target or, for the case of room temperature, a water or polyethylene moderator. The optimal size for $X_{\mathrm{m}}$ was found to be about $3.5 \mathrm{~cm}$ of water, and the calculated gain over the cube water moderator is $50 \%$, as shown in Fig. 3c. This additional gain can be achieved with a smaller

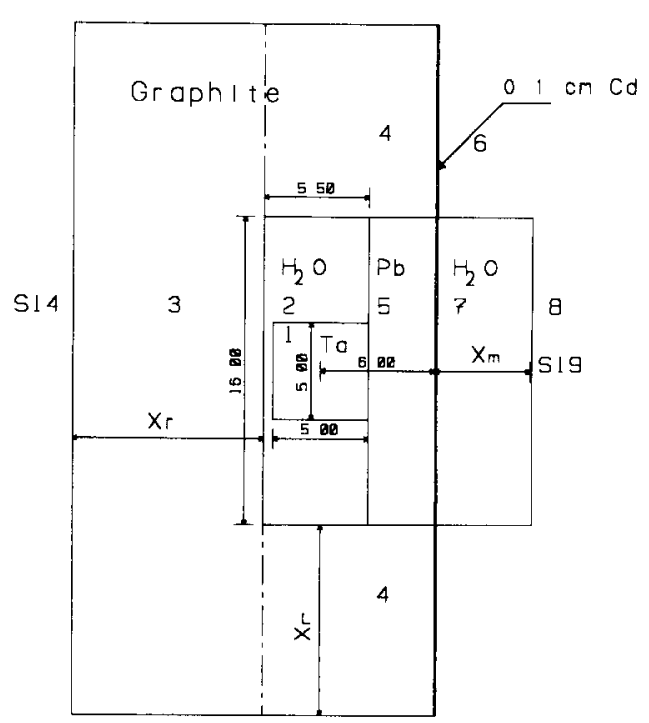

Fig. 4. The geometry used in MCNP for optımizing the reflector and moderator (cell 7) size. This geometry serves as the basis for the design of the target. (The cell numbers are shown and surfacc numbers are shown with $S$ preceding the number). 
size moderator. This case serves as the basis for a more detailed design shown in Fig. 4.

\subsection{Optimizing the reflected geometry}

The geometry in Fig. 4 shows the two moderators in cells 2 and 7 where the cold moderator in cell 7 is represented by water to compare the MCNP results to the measurements of Kiyanagi and Iwasi [5]. Cell 5 is a lead shield that reduces the gamma-ray heating of the cold moderator in cell 7. The effect of the $3.5 \mathrm{~cm}$ thick lead shield on the neutron flux was found to be a reduction of only $15 \%$ in the flux intensity while the moderator heating drops by a factor of 4 . The water moderator in cell 2 also serves as a heat sink for the lead shield in cell 5 .

In this stage of the design it was very important to compare the calculated results to some experimental results to see that the results obtained so far are correct. Kiyanagi and Iwasi [5] conducted an experiment to determine the optimum graphite reflector thickness for a slab water moderator $(10 \mathrm{~cm} \times 10 \mathrm{~cm} \times 5 \mathrm{~cm})$. The geometry used for the MCNP calculations as shown in Fig. 4 was used to calculate the optimum reflector thickness needed to maximize the neutron current emitted from the moderator face $\mathrm{S} 19$ (size of the moderator in cell 7 is $16 \mathrm{~cm} \times 16$ $\mathrm{cm} \times 5 \mathrm{~cm}$ ). The results are plotted in Fig. 5 together with Kiyangi's experimental results. These results show that a reflector size $X_{\mathrm{r}}=10 \mathrm{~cm}$ is a good choice and gives a gain of about 2 over the unreflected geometry. The results are in very good agreement with Kiyanagi's experimental results.

Kiyanagi also measured the optimum moderator thickness of a very similar geometry to the one shown in Fig. 4. To verify if our MCNP calculations agree with the measurements, the reflector thickness $X_{\mathrm{r}}$ was set to $10 \mathrm{~cm}$ and $X_{\mathrm{m}}$ (thickness of the moderator in cell 7) was varied in a series of MCNP calculations. The optimal water moderator thickness was found to be about $5 \mathrm{~cm}$. This is in good agreement with Kiyangi's experimental results that found an optimum thickness of $5.5 \mathrm{~cm} \mathrm{frr} \mathrm{a} 25 \mathrm{~cm} \times 25 \mathrm{~cm} \times$ $5.5 \mathrm{~cm}$ water moderator.

\subsection{ETT testmg}

The final target design is shown in the MCNP plot of Fig. 6. This more detailed geometry includes additional structure such as the electron beam and cooling water entry holes in the reflector and moderator and more detailed treatment of the cooling water in the tantalum target region.

The ETT was constructed according to the geometry derived from the optimization calculations, a picture of the target is shown in Fig. 7. The target included a new design for the tantalum photo-neutron target, a $5 \mathrm{~cm} \times 16 \mathrm{~cm} \times$ $16 \mathrm{~cm}$ C-shaped water moderator, a $10 \mathrm{~cm}$ graphite reflector and about $10 \mathrm{~cm}$ of lead shield surrounding the whole

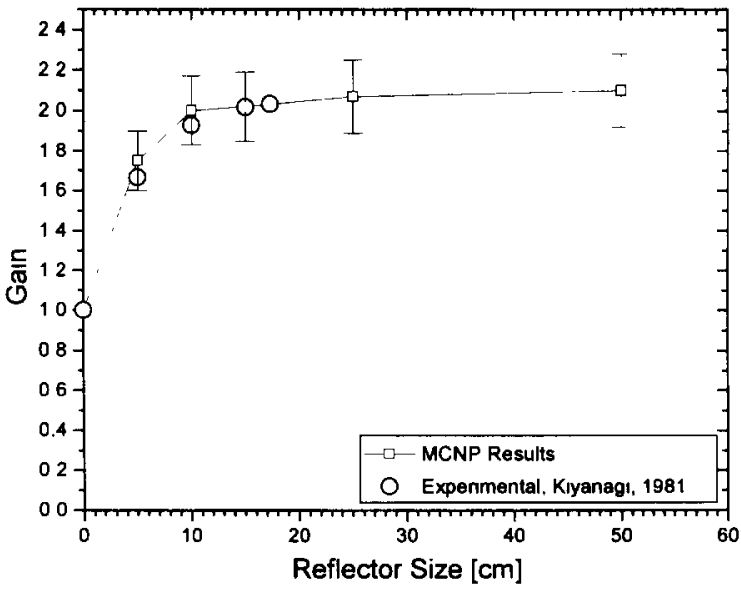

Fig. 5. Gain achieved with a graphite reflector surrounding the water moderator; the MCNP calculated results are compared to a similar experimental geometry described by Kiyanagi and Iwasi $[5]$.

structure. The target was installed in the RPI linac target room on February 1992, and various geometries were tested experimentally to achieve the optimum working configuration.

The measured ETT gain relative to the BT is plotted in Fig. 8 and compared with the MCNP calculations. In the energy range of 0.005 to $10 \mathrm{eV}$ the average calculated and measured gains are $3.0 \pm 0.4$ and $3.1 \pm 0.5$, respectively. The calculation and experiment agree in the thermal energy region from 0.005 to $0.08 \mathrm{eV}$ and from 1 to $10 \mathrm{eV}$; in the energy range between 0.08 to $1 \mathrm{eV}$ the calculation shows a higher gain. The discrepancy is in the region where the $1 / E$ slowing down spectrum combines with the thermal Maxwellian spectrum. To get more insight on the reasons for this discrepancy, the measured and calculated neutron flux shapes for both targets are plotted in Fig. 9. The calculated flux shape for the BT agrees well with the
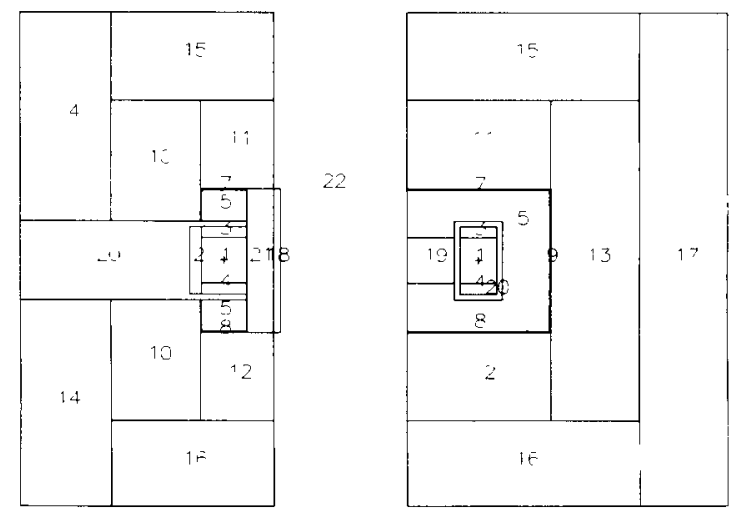

Fig. 6. The final geometry of the enhanced target as simulated by MCNP. On the left is a view from the linac and on the right is a view from the neutron flight path (the numbers shown are cell numbers). 


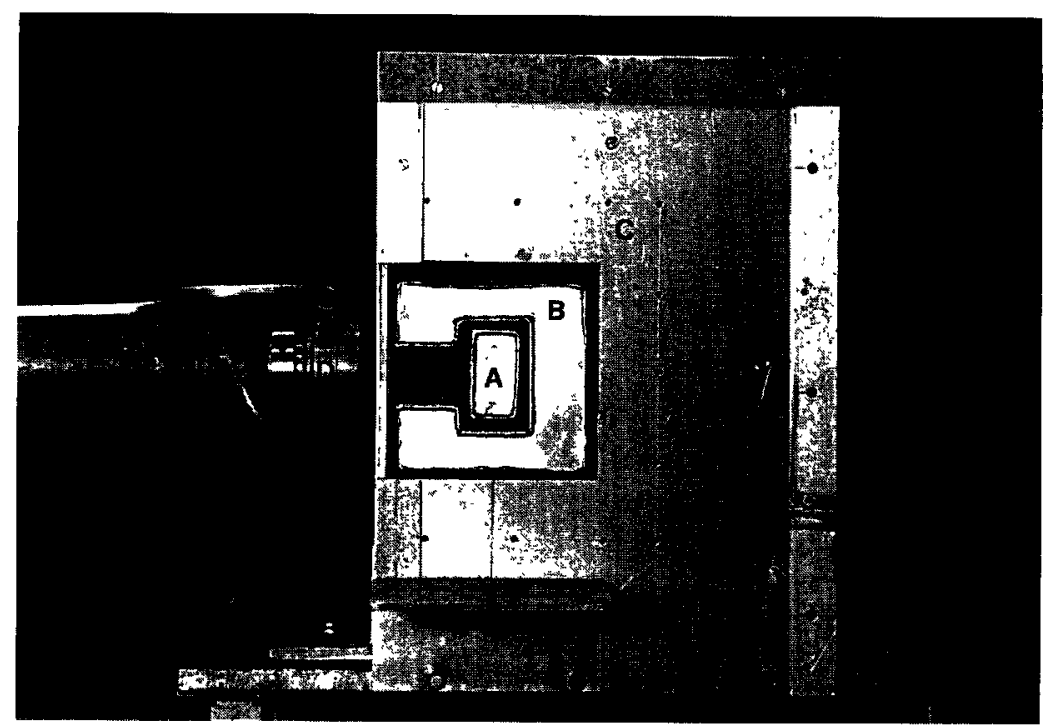

Fig. 7. A picture of the ETT showing: (a) the tantalum photo-neutron target, (b) the $\mathrm{C}$ shaped water moderator, (c) an aluminum case that contained the graphite reflector surrounded by a lead shield, (d) the linac electron beam line window.

measurements. The ETT shape shows discrepancy between about 0.1 to $1 \mathrm{eV}$, where the calculations are higher than the measured flux; thus the measured gain plotted in Fig. 8 in the same energy range is lower. A possible explanation for this discrepancy could be the fact that the lead shadow shield was not taken into account in the MCNP calculation. The shadow shield is a $20.32 \mathrm{~cm} \times 10.16 \mathrm{~cm} \times 5.08$ $\mathrm{cm}$ lead brick that is placed in front of the tantalum target to reduce the gamma-ray flux arriving at and possibly overloading the neutron detector. The shadow shield removes neutrons from the beam and thus lowers the experimental flux relative to the calculated flux. Other possible reasons could be a discrepancy between the MCNP cross section library and the actual materials used in the target structure like water and lead. Assumptions made on the

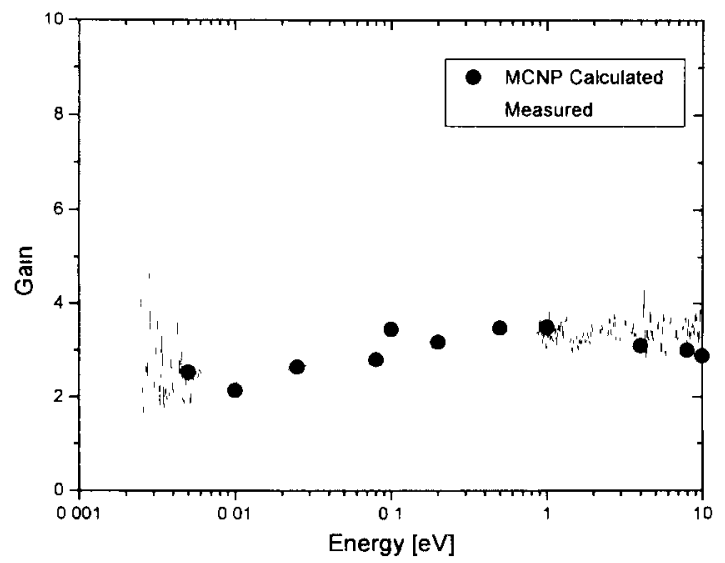

Fig. 8. Comparison of measured and calculated neutron intensity gain of the ETT (without the additional polyethylene moderator). source distribution and the tantalum target source treatment could also contribute to this effect.

Because the new target assembly was designed to be coupled to a cold moderator it is under-moderated. To further increase the gain a polyethylene moderator was placed in front of the target in the lead shield designated location (cell 21 in Fig. 6). The optimum moderator thickness was found experimentally to be about $3.8 \mathrm{~cm}$, increasing the gain by a factor of two. This resulted in the highest gain room temperature target which is an improvement of a factor of six over the RPI BT target. This increase in the neutron flux cuts the experiment time by a factor of 6 (to obtain the same counting statistics). Comparision of the signal-to-background ratios of the ETT and the BT shows

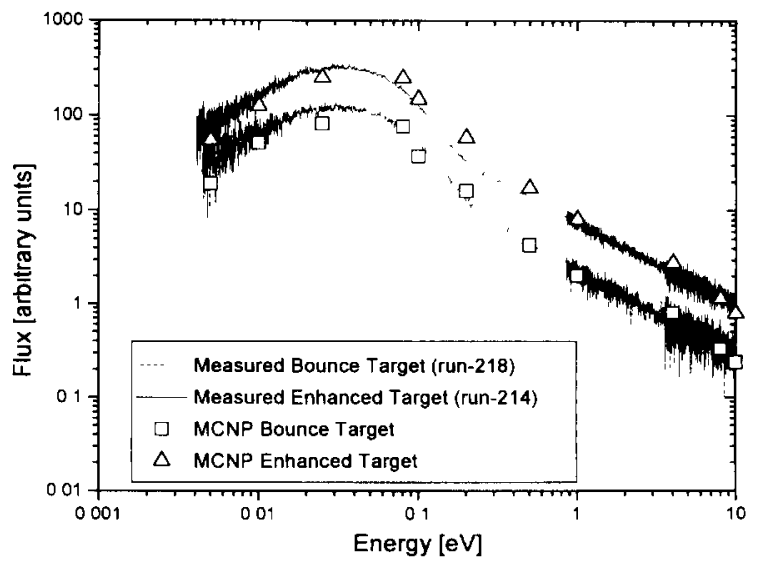

Fig. 9. Calculated and measured flux, for the BT and ETT (without the additional polyethylene moderator). 


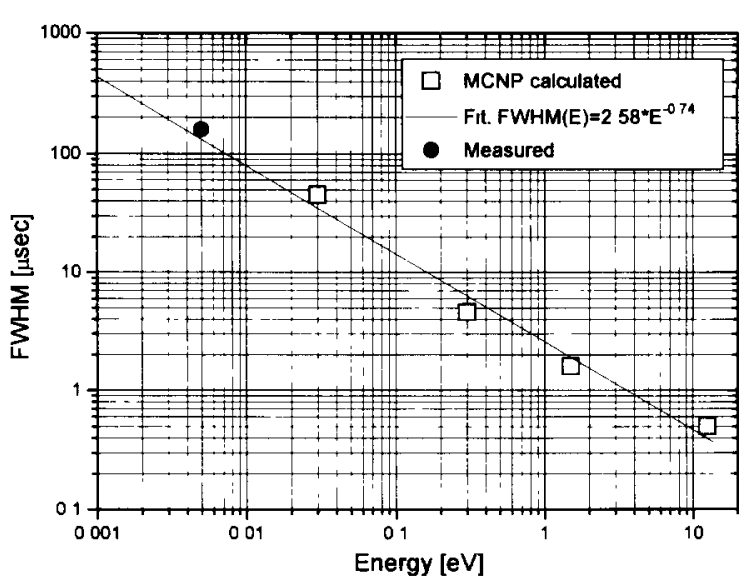

Fig. 10. Calculated and measured neutron pulse FWHM.

that below $0.1 \mathrm{eV}$ the ETT signal-to-background ratio is higher by a factor of three [2].

The ETT resolution was determined by a series of MCNP calculations that tallied the neutron current leaving the moderator as a function of energy and time [2]. The full width at half maximum (FWHM) of the neutron pulse at various energies is plotted in Fig. 10. The FWHM was fitted to a power law function and extrapolated to lower energies which were harder to calculate with MCNP. A measurement to estimate the neutron pulse width at 0.005 $\mathrm{eV}$ was performed by placing a thick (about $5 \mathrm{~cm}$ ) beryllium sample in the beam and measuring the width of the Bragg edge at $0.005 \mathrm{eV}$ at a $15 \mathrm{~m}$ time of flight distance. The width was found to be about $160 \mathrm{msec}$ which corresponds a resolution of about $1 \%$ in time-of-flight or $2 \%$ in energy. This measurement is plotted in Fig. 10 and shows good agreement with the fit to the MCNP calculations. The measured and calculated neutron pulse FWHM indicate that the resolution provided by the ETT in the thermal region is adequate for cross section measurements and can resolve Bragg structure.

\section{Cold moderator design}

In the conceptual design (Fig. 2) it was established that the cold moderator will be mounted in front (in the neutron beam direction) of the water moderator. Key parameters are the cold moderator material and thickness. The problem of finding the best material for a cold moderator is a subject of many studies (for example Refs. [6] and [7]). Moderators with high proton densities like water or polyethylene, are not effective cryogenic moderators because they have too few low frequency modes to provide for the final stages of thermalization [8]. Methane and liquid hydrogen are generally considered the best choice in terms of gain [7]. Solid methane is a better cold moderator than liquid hydrogen but it has a "burping" problem at high neutron fluence [9]. Under high neutron fluence the moderator will accumulate stored energy that can suddenly be released and cause the moderator to explode [10]. Hydrogen is liquid at low temperatures, but like methane it is highly flammable and in any problem of overheating, hydrogen gas is created and an explosion hazard exists. The RPI linac is located in a university and can not economically use any of these hazardous materials. An alternative moderator material should be considered which is safe to use and also provides high neutron flux.

There are several mechanisms of slowing down in the subthermal energy region. For a liquid cold moderator the translational motion of the moderator molecules is an important parameter in the slowing down process. A good liquid moderator would be one composed of light molecules with small intermolecular forces such as hydrogen.

For a solid cold moderator the rotational motion of molecules is an important parameter in the slowing down process. A good solid moderator should have free or hindered intermolecular rotations because these have relatively large cross sections in the sub-thermal energy range.

Utsuro et al. [11] investigated the methyl group and found mesitylene (1-3-5 trimethylbenzene, $\mathrm{C}_{9} \mathrm{H}_{12}$ ) to be an effective solid cold moderator. Mesitylene has a boiling point of $165^{\circ} \mathrm{C}$ and melting point at $-53^{\circ} \mathrm{C}$; the mesitylene [12] density as solid is about $0.91 \mathrm{~g} / \mathrm{cm}^{3}$.

Although mesitylene is solid at the operating temperature of 20 to $40 \mathrm{~K}$, the "burping" problem mentioned earlier is not of great concern for the RPI linac because of the relatively low neutron flux and the short duration of experiments (typically, 1-3 days of continuous operation).

\subsection{Cold moderator gain}

The expected moderator gain based on the measurements of Utsuro et al. is plotted in Fig. 11 as a function of the neutron energy and moderator temperature. These gains

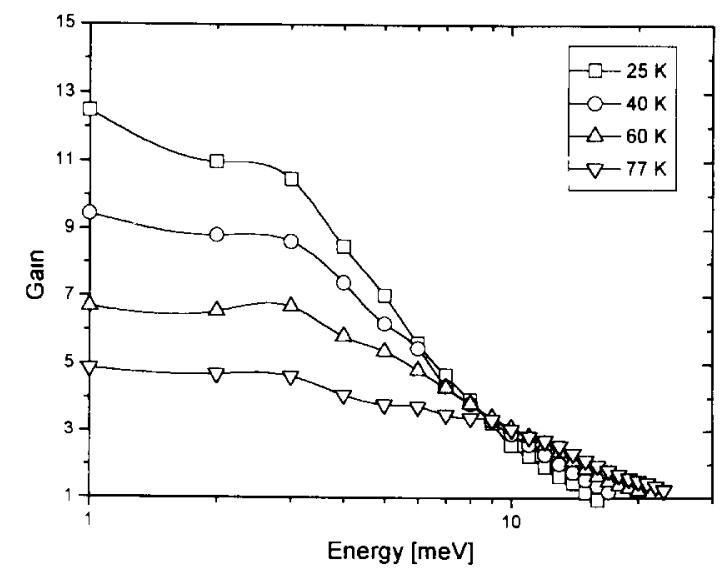

Fig. 11. Neutron spectra from a mesitylene cold moderator as a function of temperature divided by the spectrum at $280 \mathrm{~K}$. 
were calculated by dividing the measured flux at various temperatures to the flux at $280 \mathrm{~K}$. These gains show that a cold moderator will be effective only below $20 \mathrm{meV}$ and at a temperature of $40 \mathrm{~K}$ gives a gain of about 9 at $2 \mathrm{meV}$. Coupling such a moderator to the ETT results in a neutron flux about 50 times higher than the RPI BT target.

\subsection{Optimal cold moderator thickness}

When considering a transport calculation for a cold moderator, the scattering property data (scattering kernel) of the moderator at low temperature is necessary. The scattering kernel gives information about the double-differential cross section, giving the distribution in both energy and angle of the neutron after a collision. In the case of mesitylene there are no measurements of the scattering kernel at low temperatures. The only mesitylene cross section data available is the scattering cross section measured by Utsuro [13] at $10 \mathrm{~K}$.

An attempt was made to use MCNP which incorporates a free gas model to calculate the flux emerging from a mesitylene slab. The first calculations were done to see if the MCNP calculated flux has the same shape as the measured one. The results of such MCNP calculations are plotted in Fig. 12 against the spectrum measured by Utsuro et al. [11] at $40 \mathrm{~K}$. The MCNP calculation was done with a $16 \mathrm{~cm} \times 16 \mathrm{~cm} \times 3 \mathrm{~cm}$ mesitylene moderator at $40 \mathrm{~K}$ and with an evaporation neutron source spectrum with $T=0.46 \mathrm{MeV}$ (simulates the tantalum target neutrons). The results plotted in Fig. 12 show that when normalizing the two spectra at $80 \mathrm{meV}$ the MCNP calculation underestimates the thermal flux at the peak by about $30 \%$, but gives a good agreement of the overall flux shape.

The MCNP calculation does not take into account any vibrational or rotation modes in the moderator, which serve as effective slowing down mechanisms, and thus

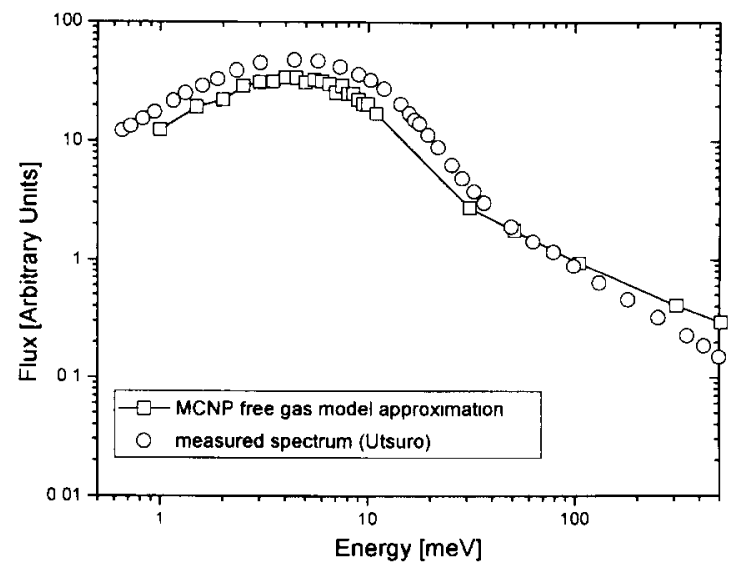

Fig. 12. MCNP calculated cold neutron spectrum compared with Usturo et al. [11] measured spectrum at $40 \mathrm{~K}$.

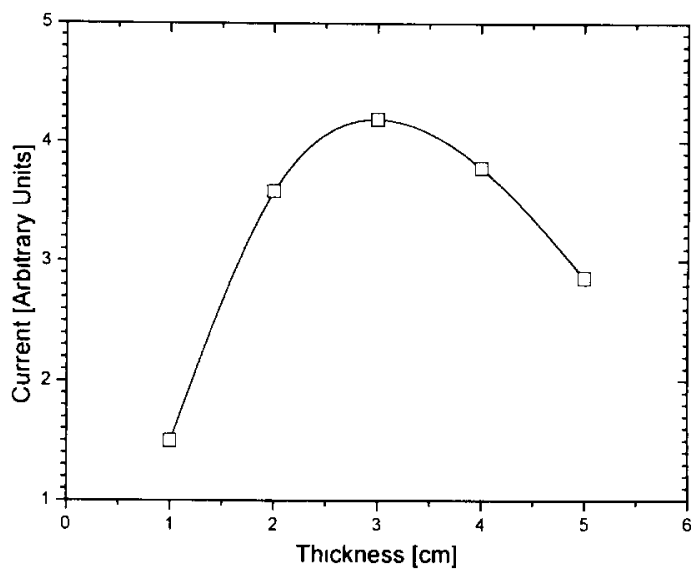

Fig. 13. Measured neutron current vs mesitylene moderator thickness (at $40 \mathrm{~K}, 16 \mathrm{~cm} \times 16 \mathrm{~cm}$ ) for incident neutron energy of 10 $\mathrm{eV}$. The measured points are connected by a spline fit.

calculates a lower flux near the thermal peak. The two flux shapes peaks are at the same energy and have about the same thermal flux shapes below $20 \mathrm{meV}$ where we expect gain from such a cold moderator. Also the $1 / E$ part of the spectrum has a steeper slope in the measurement. Overall this approximation is good enough to estimate the optimal moderator thickness needed to achieve maximum cold neutron output.

The next step was a set of calculations with variable moderator thickness. The neutron source energy was chosen to be $10 \mathrm{eV}$. The results are plotted in Fig. 13 and show an optimal moderator thickness of about $3 \mathrm{~cm}$. Clark et al. [14] made optimization calculations for their mesitylene moderator using the cross section measured by Utsuro [13] and their own Monte Carlo computer code; their optimal geometry was found to be a disk of $3.75 \mathrm{~cm}$ radius and $3.0 \mathrm{~cm}$ thick. Thus a thickness of $3 \mathrm{~cm}$ is a reasonable optimum.

\subsection{Refrigeration system requirements}

As shown in Fig. 2 the cold moderator was designed to be cooled by a cold finger connected to a cold head which is similar to the cooling method used by Clark et al. [14]. The cold moderator and finger were designed to be contained in an evacuated enclosure to reduce the heating losses by convection. In this type of system the main heat transfer mechanisms are conduction and radiation. To calculate the required refrigeration system it was first necessary to calculate the gamma-ray heating rates (the neutron heating rate is negligible) of the cold moderator structural materials. These calculations were done with the ITS [15] coupled electron photon Monte Carlo code. Using a cylindricalgeometry the target geometry could be modeled reasonably accurately. The power on the tantalum target was 
assumed to be $1500 \mathrm{~W}$, which is the typical power load expected for thermal cross section measurements. About $1300 \mathrm{~W}$ is absorbed in the tantalum target. Two cases were considered, with and without the lead shield. Of the escaping $200 \mathrm{~W}, 10 \mathrm{~W}$ will be absorbed in the cold moderator in the case with no shield and when the shield is added this number reduces to about $1.8 \mathrm{~W}$. The heating of the cold finger was found to be only about $0.6 \mathrm{~W}$. Using these values, heat transfer calculations that include radiation and conduction were performed and the cooling requirement was found to about $13 \mathrm{~W}$.

\section{Conclusions}

This paper presents the design calculations of an enhanced thermal neutron target (ETT) for the RPI electron linear accelerator. The target was optimized to generate a high thermal neutron flux to allow cross section measurements in the subthermal region. The ETT components are a 5-cm-thick C-shaped water moderator that is surrounded on five sides by a 10 -cm-thick graphite reflector, and an additional 3.81-cm-polyethylene moderator is used in front of the target to maximize the thermal neutron flux. The ETT was constructed, tested and found to deliver about six times higher flux intensity than the RPI bounce target. This allows transmission measurements to be made in the energy range from 0.001 to $15 \mathrm{eV}$ with high statistical accuracy in a relatively short time $(40 \mathrm{~h})$.

The ETT was also designed to be coupled to a mesitylene cold moderator that will further increase the neutron flux intensity by a factor of 9 in the $1 \mathrm{meV}$ energy region. Design calculations for a mesitylene cold moderator were also given. These calculations show that the optimum moderator thickness should be about $3 \mathrm{~cm}$ and the cooling requirement for the proposed system is about $13 \mathrm{~W}$ for a moderator operating at $30 \mathrm{~K}$ to $40 \mathrm{~K}$.

\section{Acknowledgments}

The authors would like to thank Mr. Jim Kelly for his valuable help in constructing the target, and for the RPI linac operators who helped mount the target and make sure it was properly coupled to the linac.

\section{References}

[1] R.W Hockenbury, Z.M. Bartolome, J.R. Tatarczuk, W.R. Moyer and R.C. Block, Phys. Rev. 178 (1969) 1746.

[2] Y. Danon, Ph.D. Thesis, Rensselaer Polytechnic Institute (1993).

[3] MCNP, A General Monte Carlo Code for Neutron and Photon Transport, Version 3B, Los Alamos National Laboratory, LA-7396-M, Rev. 2, September (1986).

[4] C.R. Stopa, Thesis, Rensselaer Polytechnic Institute (1983).

[5] Yoshiaki Kjyanagi and Hirokatsu Iwasa, J. Nucl. Sci. and Technol. 19 (1982) 352.

[6] W. Van Dingenen, Nucl. Instr. and Meth. 16 (1962) 116.

[7] J.M. Carpenter D.L. Price and N.J. Swanson, IPNS-A National Facility For Condensed Matter Research, ANL-78-88, Argonne National Laboratory, November (1978).

[8] John M. Carpenter, Cold Moderators for Pulsed Neutron Sources, International Workshop on Cold Neutron Sources, LA-12146-C, Los Alamos National Laboratory, March 5-8, (1990).

[9] IPNS Progress Report 1988-1990, Argonne National Laboratory, (1990).

[10] J.M. Carpenter, Nature 330 (1987) 358.

[11] Masahiko Utsuro, Masaaki Sugimoto and Yoshiaki Fujita, Experimental Study on a Cold Neutron Source of Solid Methyl-benzene, NA. Rep. Res. Reactor Inst. Kyoto Univ. vol. 8 (1975) 17-15.

[12] TRC Thermodynamic Tables-Hydrocarbons, Texas A and M University. Thermodynamics Research Center (1985).

[13] Mashiko Utsuro, J. Phys. C 9 (1976) 171.

[14] David D. Clark, Carol, G. Ouellet, and J. Scott Berg, Nucl. Sci. and Eng. 110 (1992) 445.

[15] J.A. Halbleib and T.A. Mehlhorn, Sandia report SAND 84-0573 (1984). 